Supporting Information

\title{
Terpolymer Multicompartment Nanofibers as Templates for Hybrid Pt Double Helices
}

Stefanie Tjaberings ${ }^{1}$, Markus Heidelmann ${ }^{2}$, Alexander Tjaberings ${ }^{1}$, Andrea Steinhaus ${ }^{1}$, Steffen Franzka $^{2}$, Bernd Walkenfort ${ }^{3}$, André H. Gröschel ${ }^{1 *}$

${ }^{1}$ Physical Chemistry and Centre for Soft Nanoscience (SoN) University of Münster, 48149 Münster, Germany.

${ }^{2}$ Interdisciplinary Center for Analytics on the Nanoscale (ICAN), University of DuisburgEssen, 47057 Duisburg, Germany.

${ }^{3}$ Institute for Experimental Immunology and Imaging, Imaging Center Essen, Electron Microscopy Unit, University of Duisburg-Essen, 45147 Essen, Germany.

Correspondence: andre.groeschel@uni-muenster.de

\section{Table of Contents}

Supporting Videos. 1

S1: TEM overview of cross-linked helix-on-cylinder morphology ................................... 2

S2: STEM dark field images of Pt@MCNFs..............................................................2

S3: Sum Spectrum of Pt@MCNFs ............................................................................................... 3

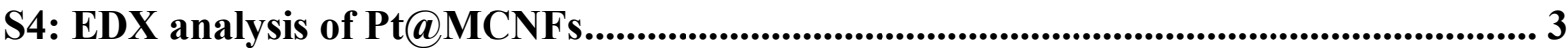

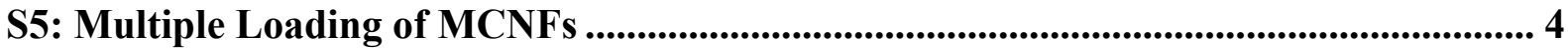

S6: Raman spectrum of Pt@C after carbonization ........................................................... 5

S7: STEM dark field images of Pt@C after carbonization............................................ 5

S8: UV/Vis spectra of MB degradation in water..........................................................6

Supporting references................................................................................................................. 6

\section{Supporting Videos}

Supporting Video 1: TEM tilt series of Pt@MCNF helix imaged in STEM mode between -60 and $+60^{\circ}$ in $1^{\circ}$ steps. The grid rotates around an $90^{\circ}$ axis.

Supporting Video 2: Reconstruction of a Pt-loaded double helical segment rotating around its $90^{\circ}$ axis.

Supporting Video 3: STEM imaging of an area of several overlapping Pt@MCNFs during the heating process in-situ from $25^{\circ} \mathrm{C}$ to $1000{ }^{\circ} \mathrm{C}$.

Supporting Video 4: Catalysis of $\mathrm{MB}$ under continuous flow using a syringe pump and a Pt@MCNF-coated $0.45 \mu \mathrm{m}$ PTFE syringe filter. 


\section{S1: TEM overview of cross-linked helix-on-cylinder morphology}

After cross-linking of the $\mathrm{PB}$ domain with $\mathrm{S}_{2} \mathrm{Cl}_{2}$ via the gas phase we observe a morphological transition. The PB shell can now be seen as single spots (Figure S1a). To verify the helical morphology, the cross-linked bulk film was redispersed in THF (Figure S1b).

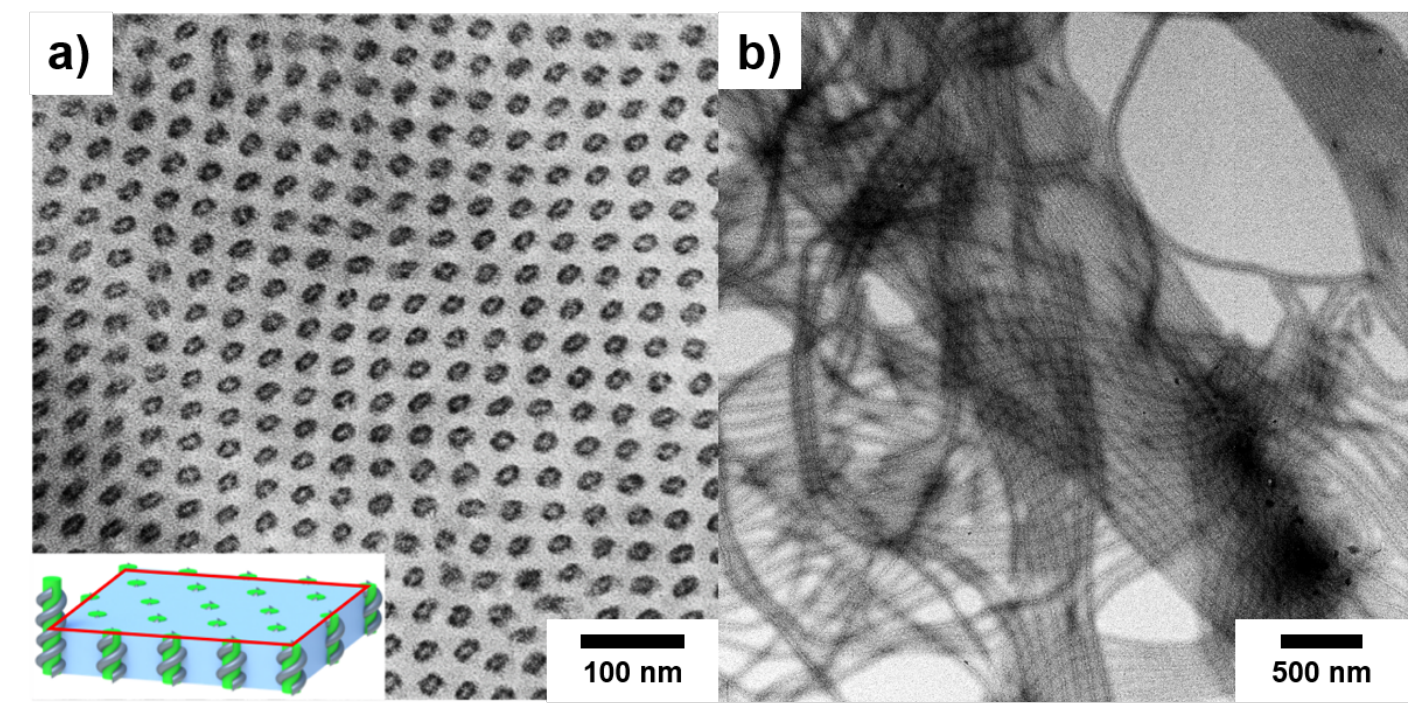

Figure S1. Cross-linked MCNF template. a) TEM of the helix-on-cylinder morphology, where the PB helix appears as dark, single spots. b) TEM overview of MCNFs after redispersion in THF (sample was stained with $\mathrm{OsO}_{4}$ ).

\section{S2: STEM dark field images of Pt@MCNFs}

The STEM images in Figure S2a shows the location of the Pt within the MCNFs and Figure $\mathrm{S} 2 \mathrm{~b}$ a close-up of the Pt double helix. The Pt double helix appears bright in dark field scanning mode.

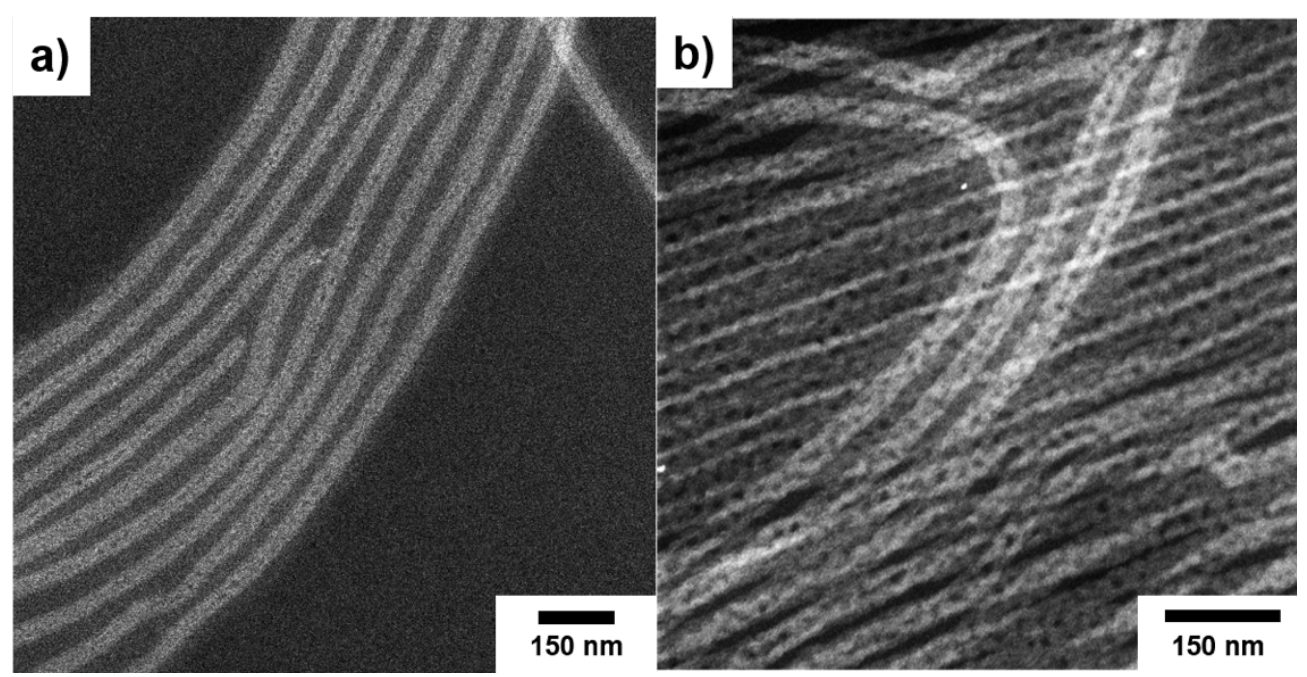

Figure S2. STEM dark field Pt double helices. a) STEM overview image and b) close-up of Pt@MCNFs. 


\section{S3: Sum Spectrum of Pt@MCNFs}

The sum spectrum in Figure S3b shows the mass percentage of oxygen, platinum and sulphur in the Pt@MCNFs.
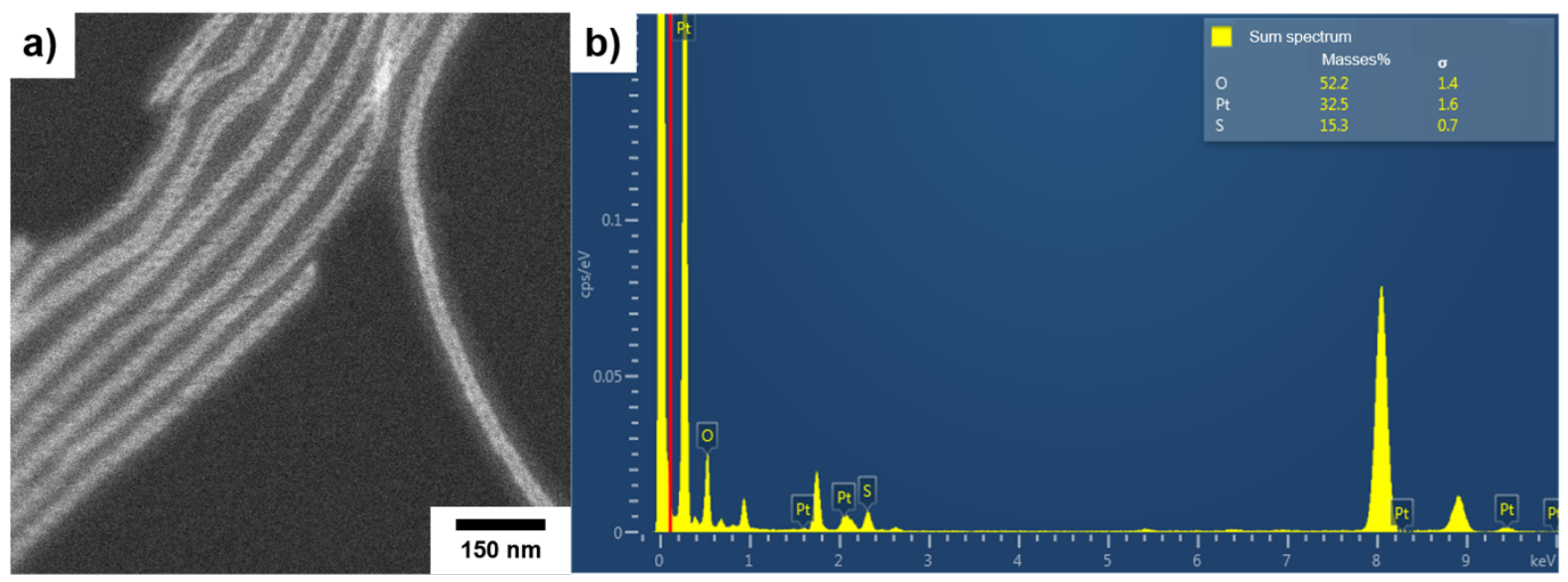

Figure S3. a) STEM dark field image of the measured area. b) Sum spectrum of Pt-MCNFs to verify the element distribution.

\section{S4: EDX analysis of Pt@MCNFs}

The EDX spectrum of the Pt double helix in Figure S4a shows the characteristic $K \alpha_{1}$-radiation of the sulphur (green pixels in Figure S4b) and the characteristic $M \alpha_{1}$-radiation of platinum (red pixels in Figure S4c). Platinum and sulphur locate in the same volume; their interaction directs the growth of the double helix.

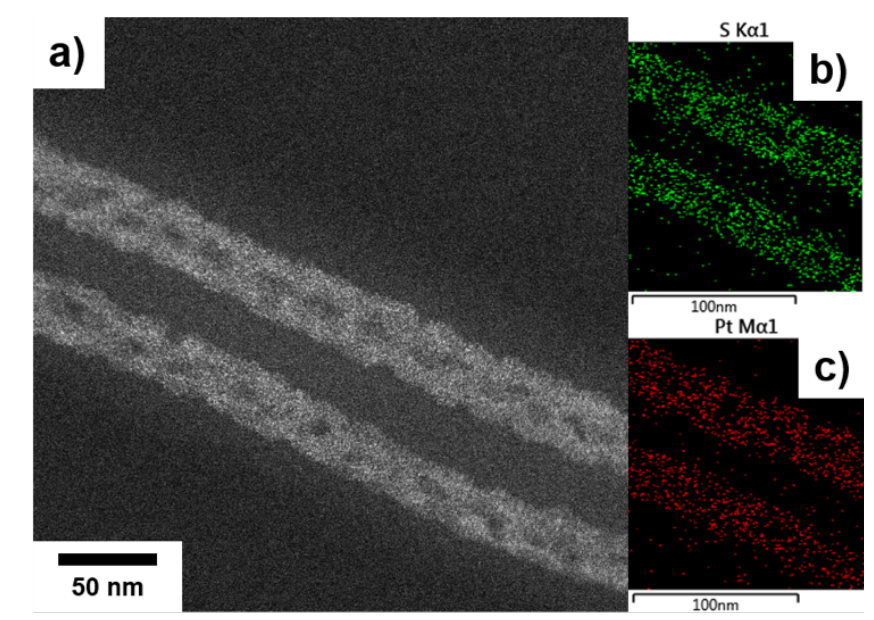

Figure S4. EDX analysis. a) STEM image of two Pt@MCNFs. EDX showing the distribution of b) sulphur (green) and c) platinum (red). 


\section{S5: Multiple Loading of MCNFs}

Figure S5 shows the results of MCNFs loaded multiple times with Pt. In the beginning, the fibers maintain their high aspect ratio and are more flexible (Figure S5a). After multiple loading cycles, the nanofibers show an increased stiffness (Figure S5b) and after the fifth loading step the fibers start to fragment into shorter rods (Figure S5 d, e).

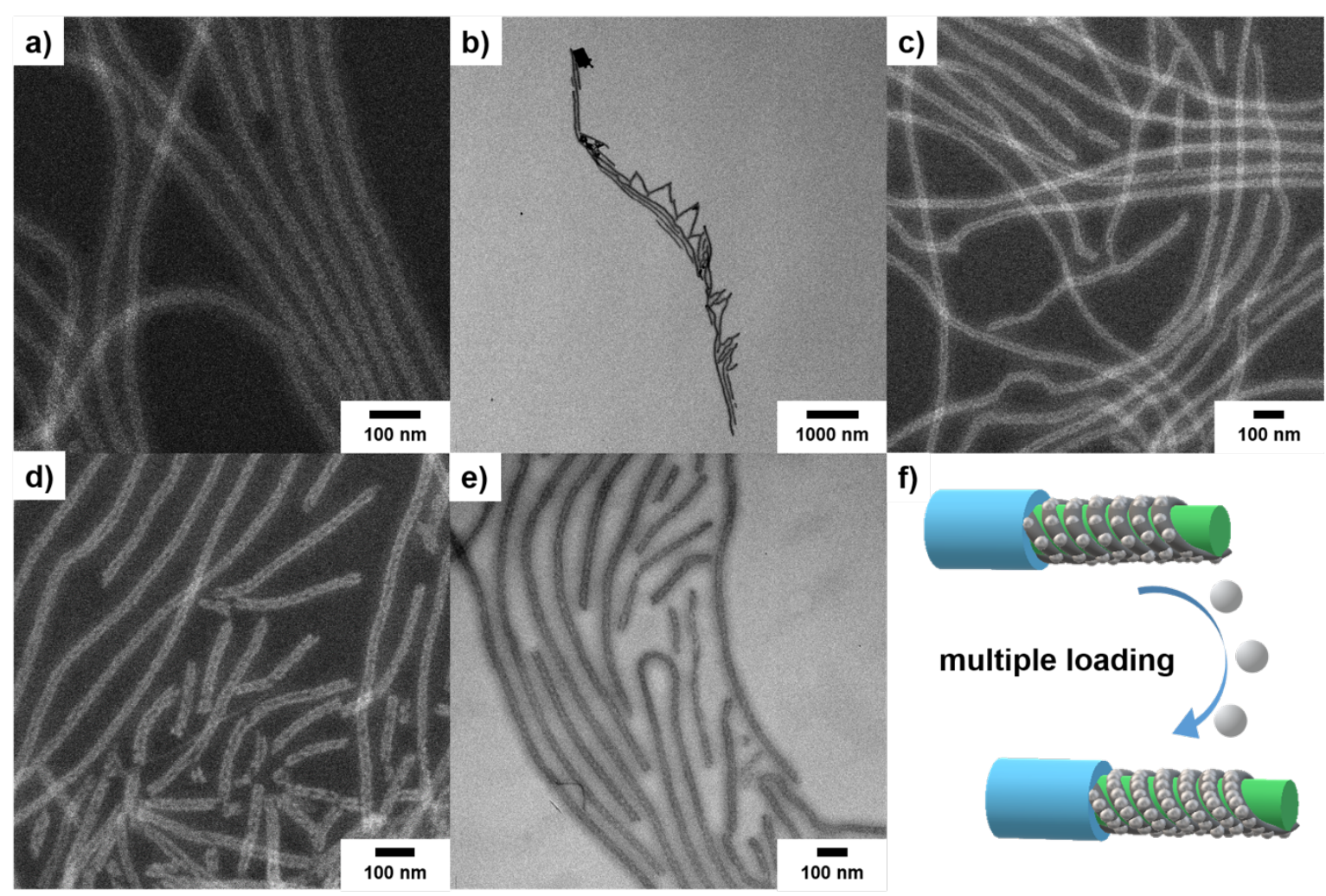

Figure S5. STEM and TEM images MCNFs after multiple Pt loading cycles. a) Single, b) double, c) triple, and $\mathbf{d}, \mathbf{e})$ fivefold loaded sample. f) Scheme illustrating increasing Pt content. 


\section{S6: Raman spectrum of Pt@C after carbonization}

The Raman spectrum in Figure S6 of a carbonized sample shows a D peak centered at $1360 \mathrm{~cm}^{-}$ ${ }^{1}$ and a $\mathrm{G}$ peak at $1582 \mathrm{~cm}^{-1}$ typical for amorphous carbon containing nanocrystalline $\mathrm{sp}^{2}$ clusters. $^{1}$

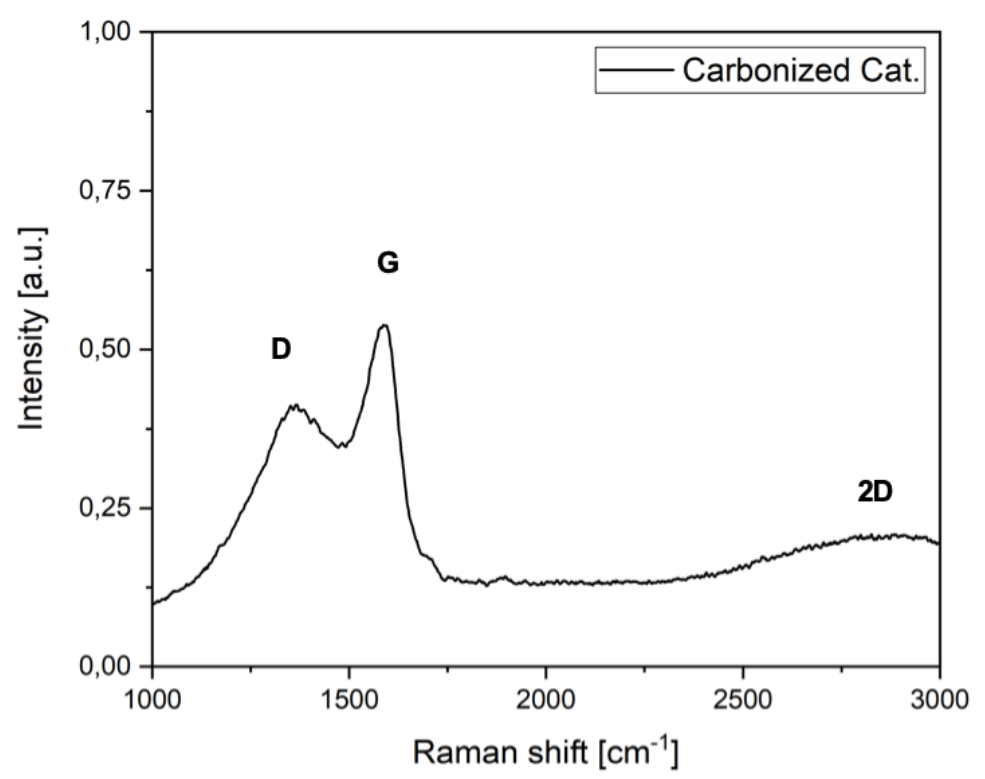

Figure S6. Raman spectrum of Pt@C sample.

\section{S7: STEM dark field images of Pt@C after carbonization}

The STEM images in Figure S7 shows Pt@C after carbonization and redispersion in THF. After heating the sample up to $500{ }^{\circ} \mathrm{C}$, the cylindrical structure of the carbonized polymer is still visible (Figure S7a) and Pt-NP are still located on the nanofibers (Figure S7b).

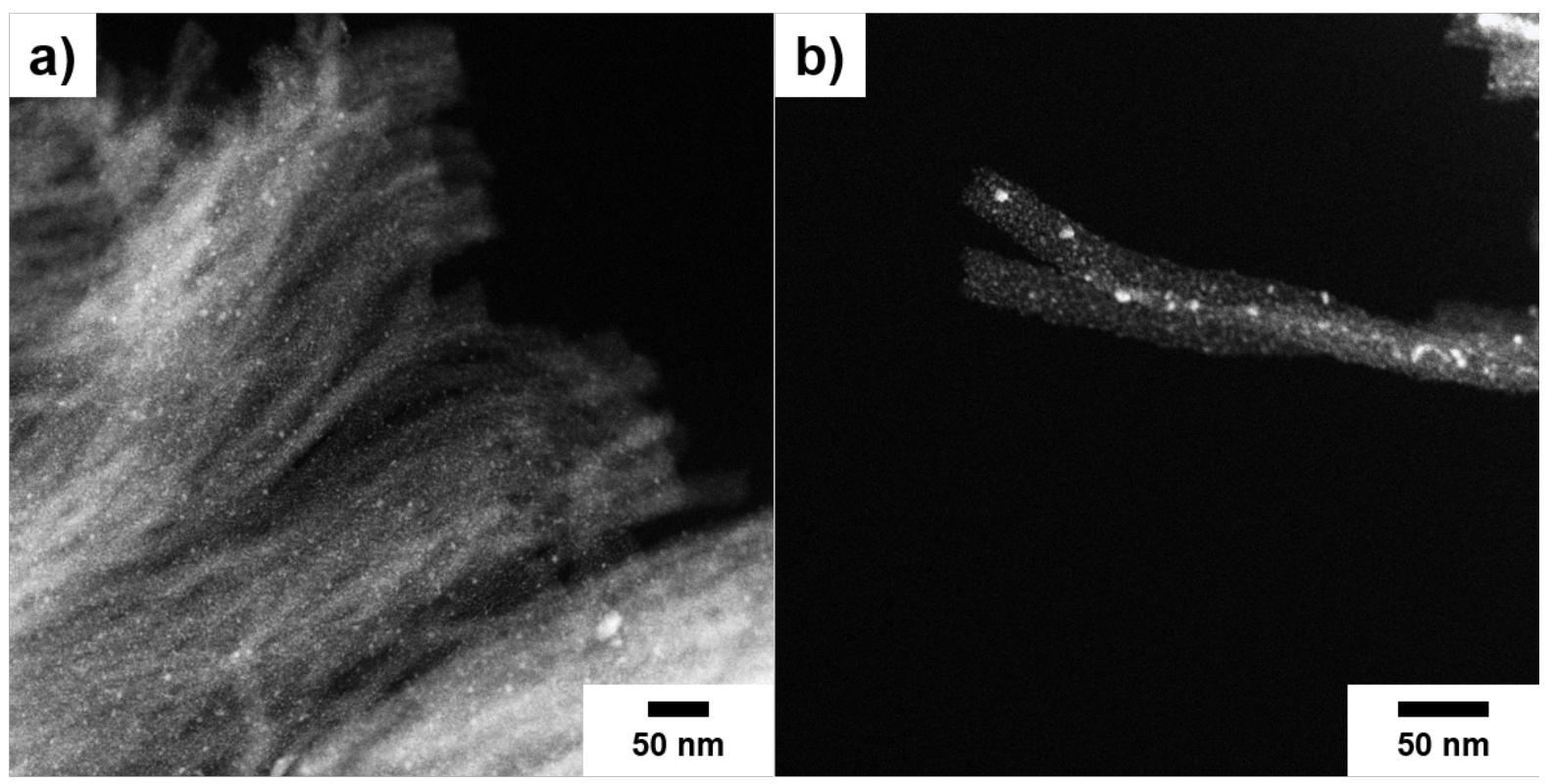

Figure S7. STEM images of Pt@C. a) STEM dark field image and b) close-up of carbonized Pt@MCNFs. 


\section{S8: UV/Vis spectra of MB degradation in water}

Figure S8a shows pure MB in water at the beginning and after $720 \mathrm{~s}$. The pure MB solution remains blue, addition of $\mathrm{NaBH}_{4}$ slightly degrades the $\mathrm{MB}$ dye (Figure $\mathrm{S} 8 \mathrm{~b}$ ). The four $\mathrm{UV} / \mathrm{V}$ is spectra in Figure S8c-f summarize the catalytic conversion of MB in water using $10 \mu \mathrm{g}, 12.5$ $\mu \mathrm{g}, 25 \mu \mathrm{g}$ of sulfonated Pt@MCNFs and $100 \mu \mathrm{g} \mathrm{Pt} @ \mathrm{C} ;[\mathrm{MB}]=2.0 \times 10^{-5} \mathrm{M},\left[\mathrm{NaBH}_{4}\right]=2.0 \mathrm{x}$ $10^{-2} \mathrm{M}$.

a)

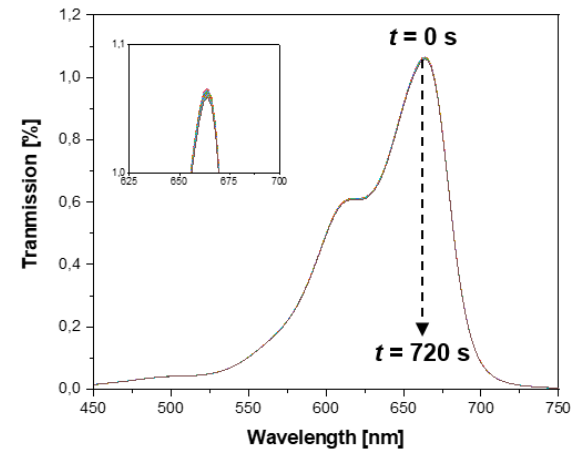

c)

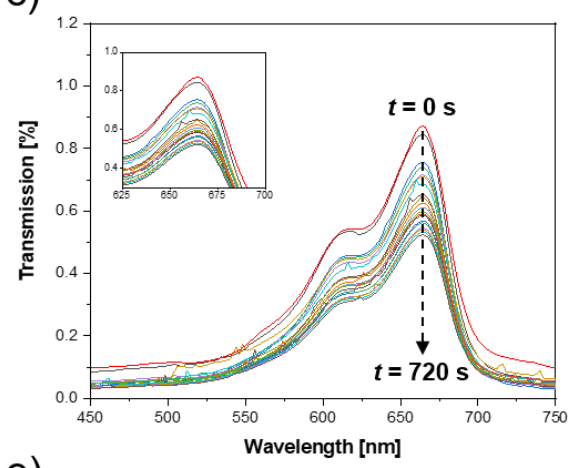

e)

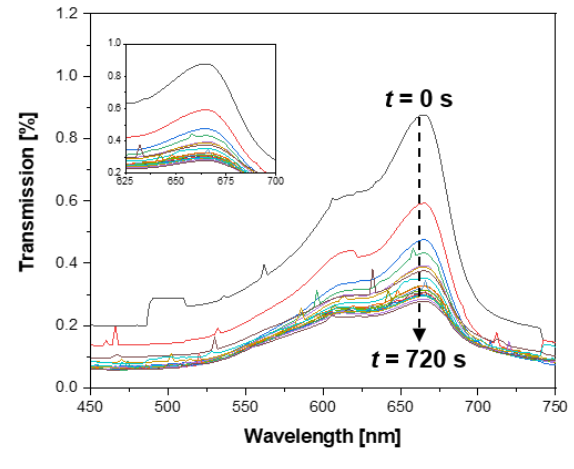

b)

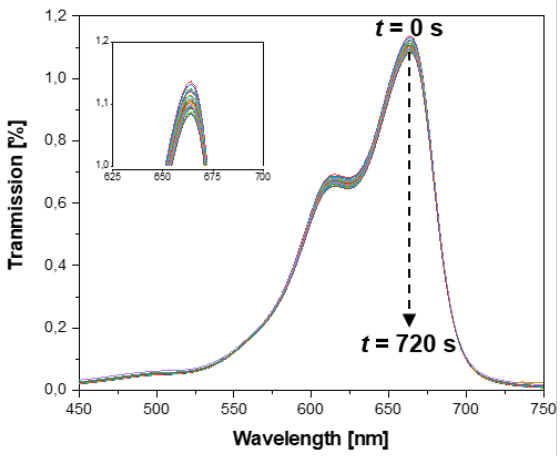

d)

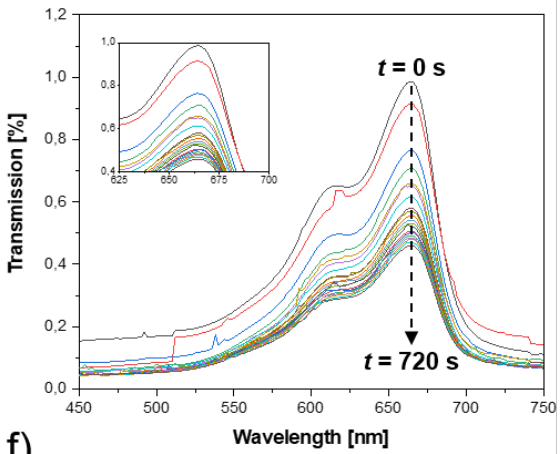

f)

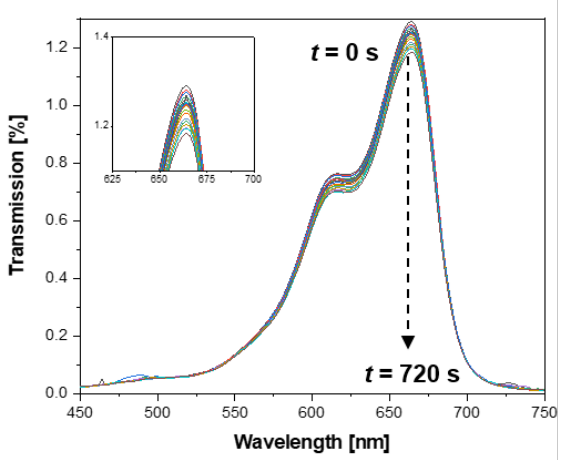

Figure S8. UV/Vis spectra of catalytic reduction of $\mathrm{MB}$ in water. a) $\mathrm{MB}$ in water. b) Autocatalytic reaction of $\mathrm{MB}$ and $\mathrm{NaBH}_{4}$ in water. c-e) $\mathrm{MB}$ degradation in water catalyzed by $10 \mu \mathrm{g}(\mathrm{c}), 12.5 \mu \mathrm{g}(\mathrm{d})$, and $25 \mu \mathrm{g}$ (e) of Pt@MCNFs, and (f) $100 \mu \mathrm{g}$ of Pt@C. Initial concentrations of $[\mathrm{MB}]=2.0 \times 10^{-5} \mathrm{M}$ and $\left[\mathrm{NaBH}_{4}\right]=2.0 \times 10^{-2} \mathrm{M}$.

\section{Supporting references}

(1) Chu, P. K.; Li, L. Characterization of Amorphous and Nanocrystalline Carbon Films. Mater. Chem. Phys. 2006, 96 (2-3), 253-277. 